\title{
Adaptive Control Technique Effects on Single Link Bilateral Articulated Robot Arm
}

\author{
Nuratiqa Natrah Mansor ${ }^{1}$, Muhammad Herman Jamaluddin ${ }^{2}$, Ahmad Zaki Shukor ${ }^{3}$ \\ Faculty of Electrical Engineering, Universiti Teknikal Malaysia Melaka (UTeM) \\ Durian Tunggal, Melaka, Malaysia
}

\begin{abstract}
This paper describes a technique for addressing the issue of instability within force controller by developing a model of a bilateral master-slave haptic system that incorporates a Disturbance Observer (DOB) in a robotic simulation. The suggested modeling is used in conjunction with conventional controllers to be correcting undesired noise that occurs inside the working system of a particular joint of the youBot arm. To acquire the target position, the controller will additionally compensate for interference by changing its position response. Two tests were carried out to examine and compare the system's feedback that employed the proposed approach and another system with the conventional and standard-setting. The experimental findings demonstrate the resilience of the suggested system, as the system integrated with observers is more precise and faster. All of the system feedbacks from conducted experiments are measured in the simulation platform.
\end{abstract}

Keywords-Force and position controller; disturbance observer; simulated bilateral system; adaptive control; manipulator arm

\section{INTRODUCTION}

In technology pieces of machinery, a bilateral control system and autonomous robot is cutting-edge technology that allows humans to engage with situations that are inaccessible to them, owing to that the location is situated distant or dangerous. Because the robot is small-scale in size and adaptable, it is simple to control and moved freely and easily. At the same time, it also transfers the haptic sense of an isolated place. Studies in [1] reviewed that this automation has been utilized in a variety of sectors, including telesurgery, autonomous teleoperation for sea and space operations as well as dealing with explosive or high radiation operations. Since the year of 1980, haptic technology has expanded and numerous researchers have explored studies on many parts including its working control system, machine learning, architectures, communication, and applications in many landscapes [2].

Regardless of how the bilateral control system's functionality is proved to behave superiorly with directly corresponds to its pair of manipulators or devices, it is vital to maintain its high feedback gains in both position and force control for acquiring transparency. Typically, to measure the amount of torque acting upon the motor of the manipulator link, force sensors are responsible for doing the calculations.

However, the use of traditional approaches such as force sensors as part of the component as mentioned in [3] was appeared to be some limitations and disadvantages for the running system. Aside from the limited shelf life and costly price, it brings certain uncertainties, instability, and delays to the operating control system, including in robots [4]. Plus, to optimum the budget for purchasing the sensors, most works and studies focusing on upgrading the operationality of a premade assistive device like electronic joysticks, keyboards, data gloves, and custom-built manipulators [5]. These input devices communicate its direction and tasks of various aspects to the device it is controlling but never experiencing two sides tactile perceptions aside from its unilateral common vibration feedback based on onscreen actions.

On the other hand, a simpler approach for a better framework of bilateral control system can be attained by using Disturbance Observer (DOB). From past studies, the control action and feedback inside a bilateral control system may be improved up to $90 \%$ inaccuracy, compared to the standard system without DOB. As records, [6] observed that the prior system suffered around $25 \%$ to reach the ideal efficiency value. Following the implementation of DOB, contributes to the infallibility of the control process simultaneously and improving the response process within the system. This outturn should be prioritized in designing a function-based controller design deal for a bilateral control system. Plus, this control technique is practical and offers better capability as shown in [7] to the telerobotic and robot manipulator control system which has been validated and proved in past research.

Considering the notion of programmable task handling and knowledge in controller design, this study presented an adaptive approach for bilateral control system design for industrial arm robots. KUKA youBot is chosen as the manipulator and also interfaces for creating a model of bilateral master and slave robotic control system as it has open interfaces that can use for various experiments and also a mobile industrial manipulator. Furthermore, the integration of adaptive design of force and position controller into the robotic arm bilateral system is expected to demonstrate the disparity and uniqueness when operating in an industrial robotic arm, in contrast to smaller and common assistive devices like being studied in [4] and [8].

In summary, the purpose of this work is to demonstrate the proposed idea of implementing the DOB technique into a bilateral master-slave control system and its relations with an industrial robotic arm. Section 2 goes into further detail on the approach and procedure used to carry out the experiments. Section 3 will show the data and information gathered from the experiment, which has been tabulated and plotted into multiple graphs. Following that, the feedback of the stated

Zamalah Scheme, Universiti Teknikal Malaysia Melaka (UTeM) 
system from the simulation of the bilateral master-slave arm manipulator is reviewed in Section 4. While in Section 5, conclusions and recommendations for forthcoming works are commented here. The study presented in this paper is limited to provide a way to design an adjustable system that is capable to improve the complexity and effectiveness of the bilateral control system on a robotic arm manipulator with a type of robust control tool (DOB).

\section{METHODOLOGY}

The whole workflow for this study can be visualized in diagram Fig. 1 below for a clear understanding of the work process and to illustrate the step-by-step activities.

\section{A. Modeling for Disturbance Observer (DOB)}

The primary objective of this project is to use a software simulation to create a simpler framework of a bidirectional master-slave haptic system on the industrial robotic arm. As discussed priorily in the introduction, a close loop system response carries noise and experiences delay in its operation. The robustness and sensitivity of the system are typically subpar and difficult to maintain. To address such an issue, an idea for incorporating a robust control tool into the close loop control systems is required. By eliminating uncertainty and undesirable information within the system, DOB implementation might increase system infallibility. Additionally, this technique facilitates robot navigation and task manipulation between the operator and target objects. Also, force measurement through a force sensor can be substituted with this observer due to the sensors' notable drawbacks [7]. On the other hand, using conventional controllers for the control system is insufficient due to certain limitations.

In comparison to these common controllers, proportional derivative (PD) is very suitable to combine with DOB when designing a new framework control system for the bilateral robotic arm. In many engineering applications, DOB is widely approached in absence of a force sensor. Commercial force sensors are practically less robust, not cost-effective, and have limited bandwidth for sensing [8]. Hence, this intuitive robust control technique can estimate the disturbance force, $F_{d i s}$, and calculate the compensatory current, $I_{c m p}$, required to achieve robust motion control. Information estimated from the observer is used in conjunction with the input signal as feedback to reject any instabilities and achieve robust stability of providing high-accuracy readings for accurate tracking. To that end, the analogous system for (1) is depicted as a system block diagram in Fig. 2 and Fig. 3 below. $F_{d i s}$ is composed of the following components:

$\mathrm{F}_{\text {dis }}=\mathrm{F}_{\text {ext }}+\mathrm{F}_{\text {int }}+\mathrm{F}_{\text {fric }}+\left(\mathrm{M}-\mathrm{M}_{\mathrm{n}}\right) \mathrm{s}^{2} \mathrm{X}_{\mathrm{res}}+\left(\mathrm{K}_{\mathrm{tn}}-\mathrm{K}_{\mathrm{t}}\right) \mathrm{I}_{\mathrm{a}}^{\mathrm{ref}}$

The disturbance force, $F_{d i s}$ equation is the result of a modeling error in the nominal mass, $M_{n}$, and thrust coefficient, $K_{t n}$. Under interactive force, $F_{\text {int }}$, the components examined are Coriolis term, centrifugal term, and gravity term. As a consequence, $F_{\text {dis }}$ is calculated in the following manner using the low pass filter (LPF):

$\mathrm{F}_{\text {dis }}=\frac{\mathrm{g}_{\text {dis }}}{\mathrm{s}+\mathrm{g}_{\mathrm{dis}}} \mathrm{F}_{\mathrm{dis}}$

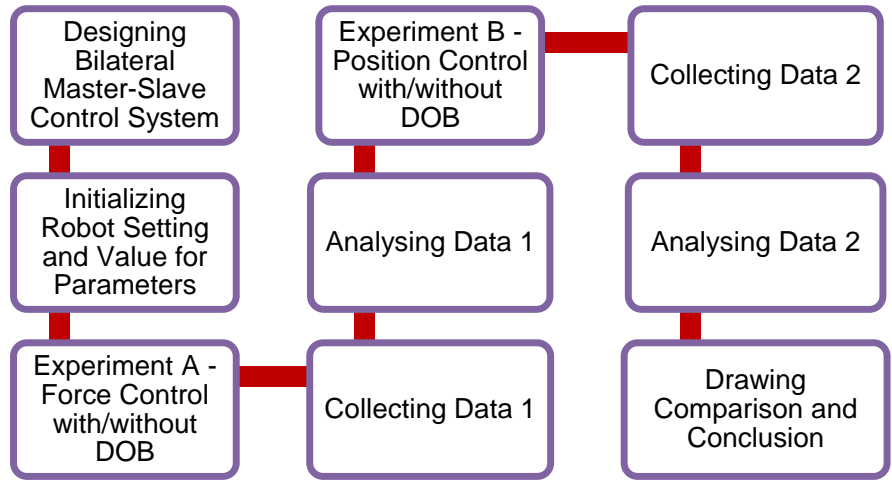

Fig. 1. The Work Process for the Study.

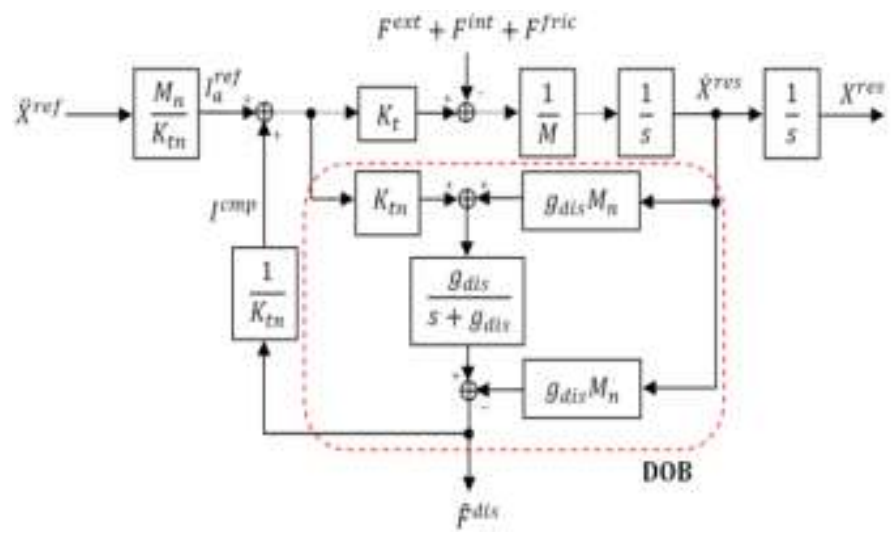

Fig. 2. Disturbance Compensation by DOB.

To put it another way, DOB assists the system in achieving solid acceleration control. Fig. 3 illustrates the block diagram of an acceleration-based position control system.

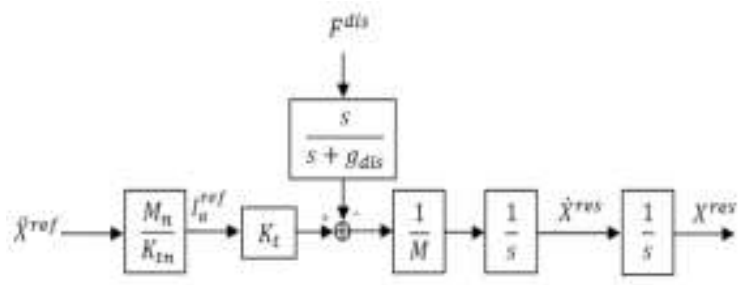

Fig. 3. Robust Acceleration Control.

Position controller, $C_{p}$ is:

$C_{p}=K_{p}+s K_{v}$

While the related position response is described as:

$\mathrm{x}_{\mathrm{res}}=\frac{c_{p}}{s^{2}}\left(x_{c m d}-x_{\text {res }}\right)$

Equation (4) has been rearranged and converted into (5) as follows:

$$
\begin{aligned}
& \frac{x_{r e s}}{x_{c m d}}=\frac{C_{p}}{s^{2}+C_{p}} \\
& \frac{x_{r e s}}{x_{c m d}}=\frac{s K_{v}+K_{p}}{s^{2}+s K_{v}+K_{p}}
\end{aligned}
$$




$$
\frac{x_{r e s}}{x_{c m d}}=\frac{2 \xi \omega_{n} s+\omega_{n}^{2}}{s^{2}+2 \xi \omega_{n} s+\omega_{n}^{2}}
$$

The natural angular frequency, $\omega_{n}$ is equal to $\sqrt{K_{p}}$ or $1 / 2 K_{v}$ whereas the damping ratio, $\xi$ can be adjusted to 1.0 to get a critical damping effect.

\section{B. Framework Design of Bilateral Master-Slave Control with DOB Based}

Following the integration of DOB into the system, the bilateral control system as a whole is illustrated in Fig. 4. The combination of the information from the said observer and the input signal produced feedback that compensates for any modeling error or interference that occurs. The total acceleration, $\ddot{x}_{d i f}$ for position controller $C_{p}$ in Differential Mode, and total force, $\ddot{x}_{\text {com }}$ for the force controller $C_{f}$ in Common Mode, are equated as in (6) and (7) for this master and slave bilateral paradigm.

$\ddot{\mathrm{x}}_{\text {dif }}^{r e f}=C_{p}(s)\left(x_{s}^{r e s}-x_{m}^{r e s}\right)$

$\ddot{\mathrm{x}}_{\text {com }}^{\text {ref }}=C_{f}(s)\left(f_{s}^{e x t}-f_{m}^{e x t}\right)$

\section{Simulation and Experiment Setup}

The work platform for this project is robotic simulation software. To determine if the proposed system is ideal and follows the rule of action and reaction in bilateral control systems, the simulation is performed in the Virtual Experimentation Platform (VREP), a 3D Robot Simulation Software with beginning parameters pre-configured similarly to in the actual environment. VREP software includes a preconfigured KUKA youBot.

The scene, models, and object attributes included therein are simple to manage and have a plethora of choices to be controlled and numerous functionalities. To implement the system's bilateral communication with a DOB-based robust control technique, KUKA youBot incorporates a model of the bilateral master-slave control system, as seen in Fig. 5.

Both the Python command script and the remote Application Programming Interface (API) function are used to link the two robots to the system. Although youBot is a mobile robot with five ${ }^{\circ}$ of freedom and has multiple links, this study focused only on 'Joint0' (the first joint located on the lower robot component). This is because to decrease the complexity of operating the haptic test and speed up the time required operating further joints and trajectory movement. Essentially, the setup and workstation for a bilateral master-slave robotic arm have been modeled in VREP. Fig. 6 illustrates the perspective view of the robot setup and environment utilized in each experiment.

In general, both systems and robot settings are equivalent. Constant variables such as the starting position of Joint 0 of both robots are fixed at $0^{\circ}$. The obstacle's mass placed alongside the slave is set to $500 \mathrm{~N}$. Throughout the simulation's execution for all experiments, the procedure is specified in flowchart Fig. 7 and presented in Fig. 8 and 9.
In summary, this section discussed the modeling designations for the specified controller and introduced observer to the close loop system, new block diagrams for bilateral control master-slave system, and robot setup in VREP simulation staged for further experiments. Procedures and workflow for experiments have been described to provide insight into how the tests are conducted.

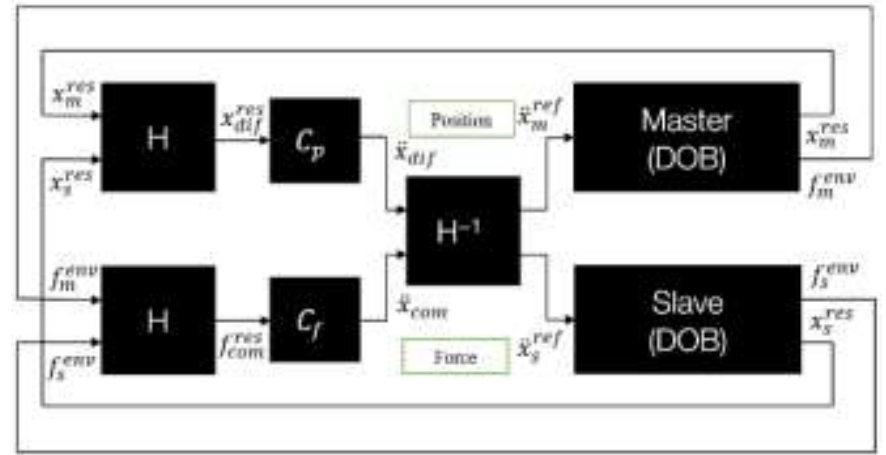

Fig. 4. Block Diagram of the Bilateral System with the Construction of DOB.

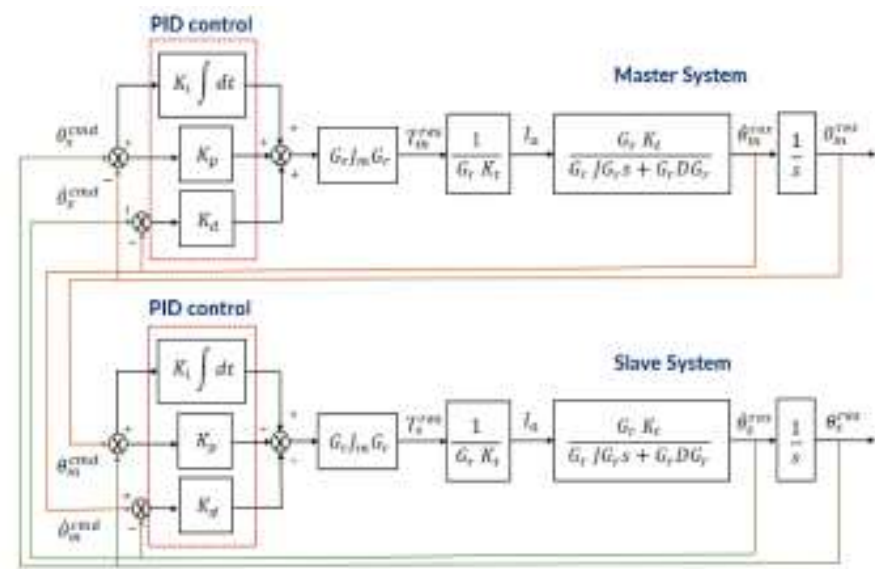

Fig. 5. Block Diagram of Master-Slave Control System with PID Controller.

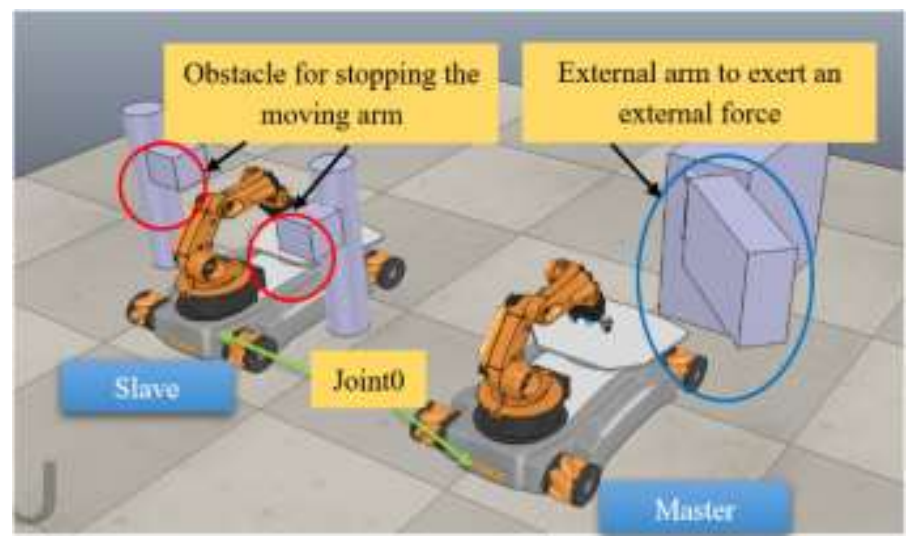

Fig. 6. Bilateral Robot Setup from Perspective view for Experiments. 


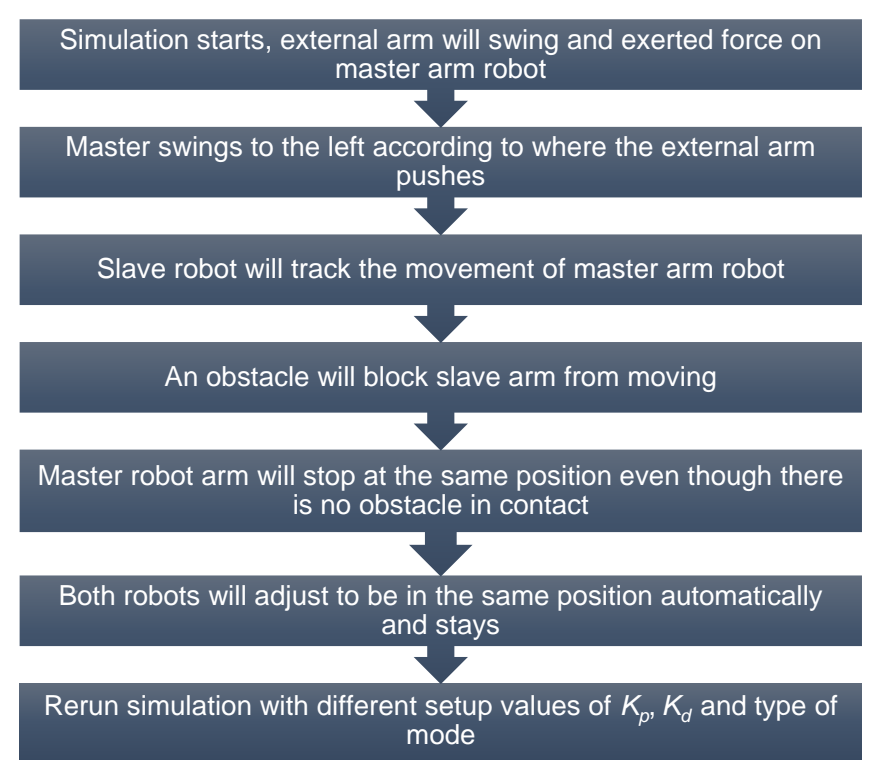

Fig. 7. Steps in the Operation of Bilateral Control System Experiment.

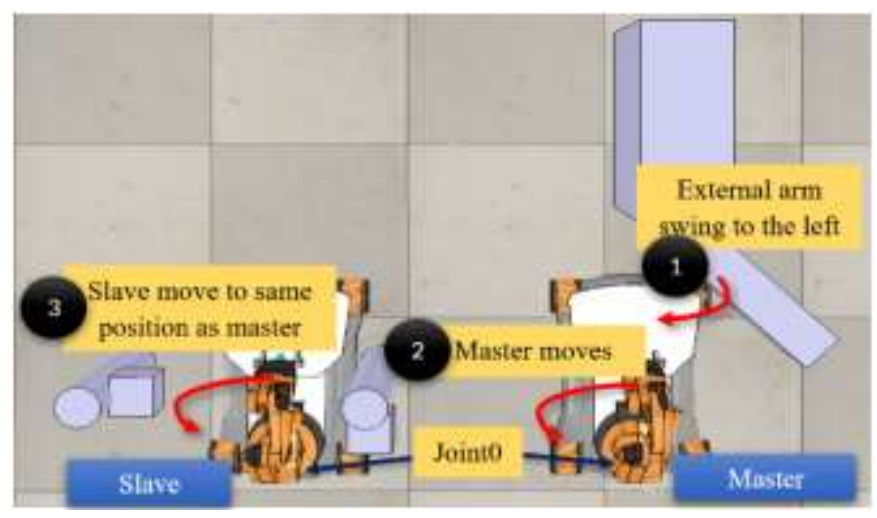

Fig. 8. Illustration A for Bilateral System Operation.

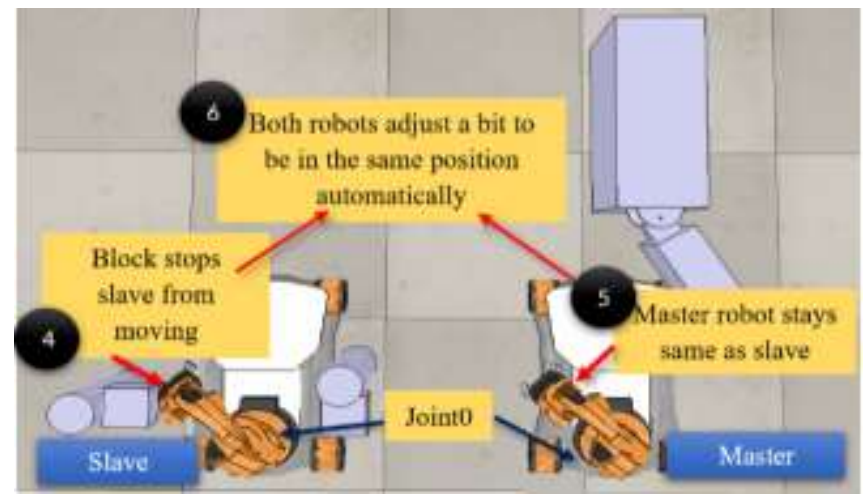

Fig. 9. Illustration B for Bilateral System Operation.

\section{ReSUlt AND ANALysis}

To demonstrate the second law of bilateral control system which is a total difference of position reading in master and slave must be equal to zero, the experiment was solely focused to prove on the differential mode law. Next, the purpose of this experiment is to observe whether the input feed carried by DOB can be used to improve the system's performance or reducing noise that occurs internally. PD controller is also paired with DOB for the proposed system.

The findings from the simulation are portrayed in this section. Response from the DOB-based control system for the articulated bilateral arm manipulator is shown in this subsection in the form of tables and graphs. Each experiment is repeated three times with a different value set of variables. $K_{p}$ and $K_{d}$ are independent variables while the initial value of the robot joint is the controlled variable. Values for independent variables are shown in Table I below. These parameters are chosen using an experimental validation technique that involves selecting a range of the best acceptable values from a large number of trials ranging from low to high. Every value of variables was necessary to repeat thrice to get the mean value before plotting into graphs accordingly.

TABLE I. $\quad \Omega_{\mathrm{N}}, \mathrm{K}_{\mathrm{P}}$ AND $\mathrm{K}_{\mathrm{D}}$ VALUES FOR DOB EXPERIMENT

\begin{tabular}{|l|l|l|}
\hline$\omega_{n}$ & $K_{p}\left(\omega_{n}\right)^{2}$ & $K_{d}\left(2 \omega_{n}\right)$ \\
\hline 1 & 1 & 2 \\
\hline 2 & 4 & 4 \\
\hline 5 & 25 & 10 \\
\hline 10 & 100 & 20 \\
\hline 20 & 400 & 40 \\
\hline 50 & 2500 & 100 \\
\hline 100 & 10000 & 200 \\
\hline 200 & 40000 & 400 \\
\hline 500 & 250000 & 1000 \\
\hline
\end{tabular}

A. DOB based for Force Control (Part A)

The graphs in Fig. 10 to Fig. 18 illustrate the force readings at Joint 0 recorded for both master and slave youBot arms.

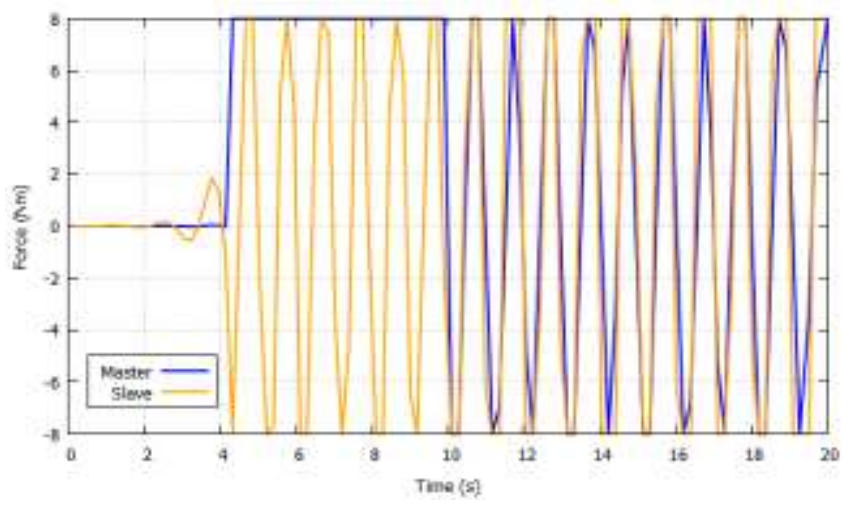

Fig. 10. Force of Master and Slave Arm vs. Time for $\omega_{n}=1$. 


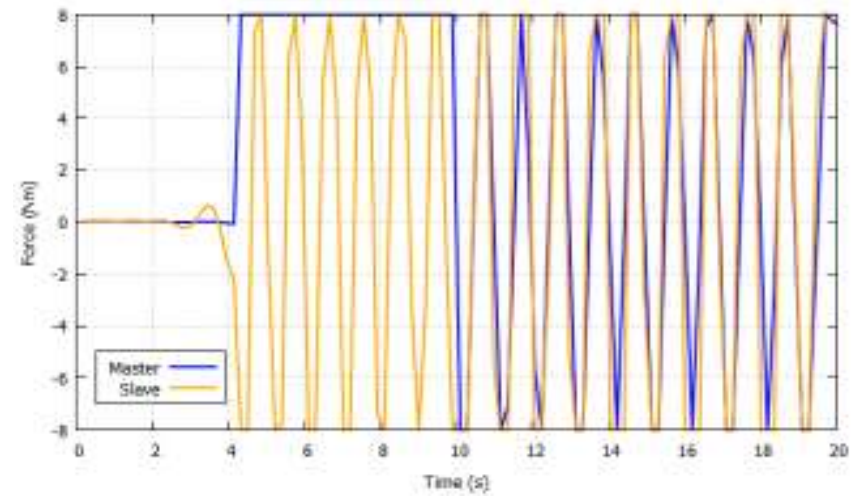

Fig. 11. Force of Master and Slave Arm vs. Time for $\omega n=2$.

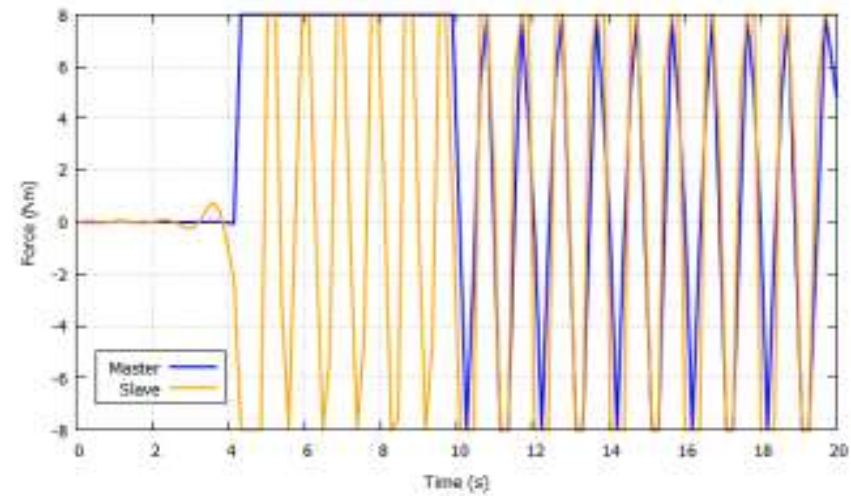

Fig. 12. Force of Master and Slave Arm vs. Time for $\omega n=5$.

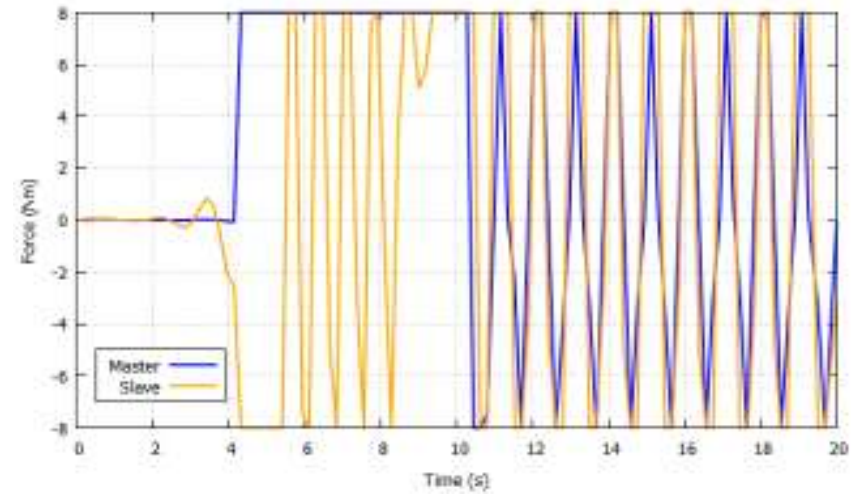

Fig. 13. Force of Master and Slave Arm vs. Time for $\omega n=10$.

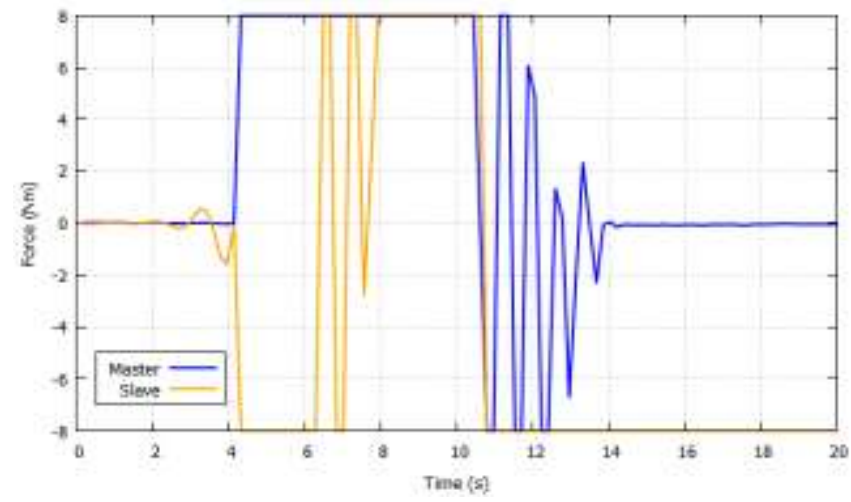

Fig. 14. Force of Master and Slave Arm vs. Time for $\omega n=20$.

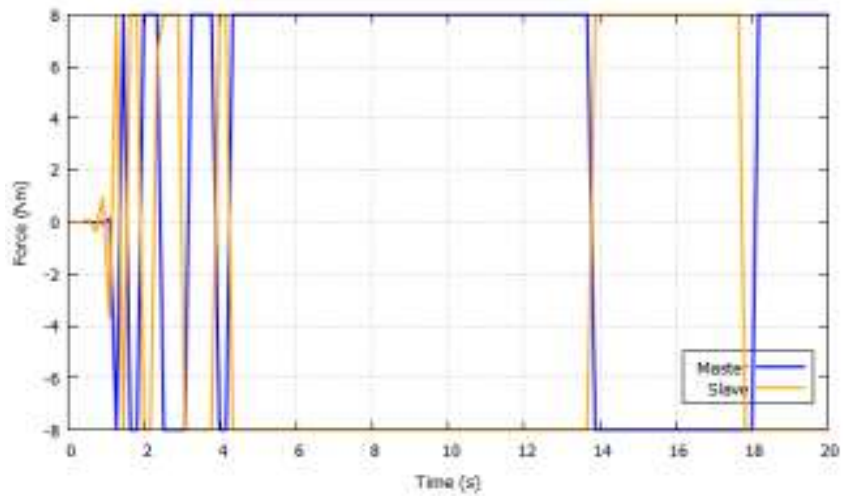

Fig. 15. Forces of Master and Slave Arm vs. Time for $\omega n=50$.

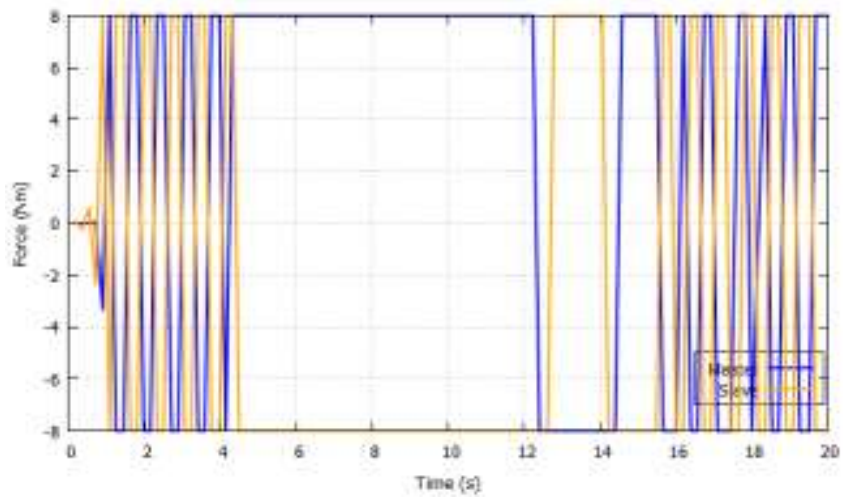

Fig. 16. Force of Master and Slave Arm vs. Time for $\omega n=100$.

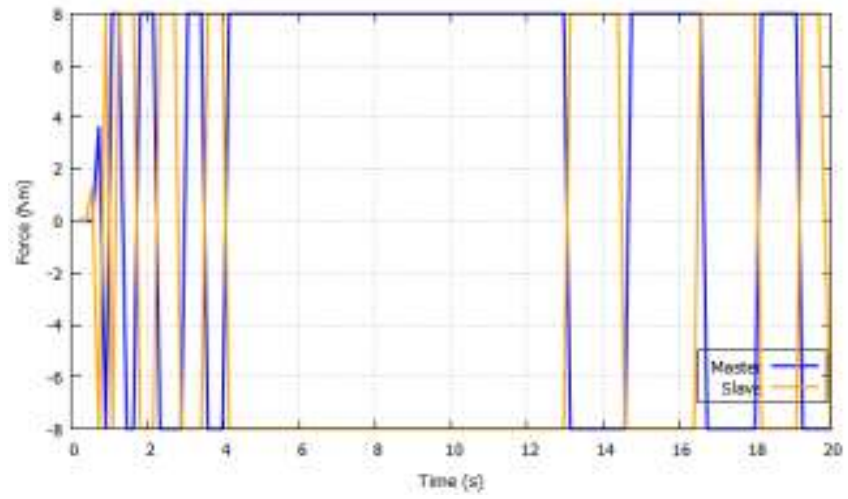

Fig. 17. Force of Master and Slave Arm vs. Time for $\omega n=200$.

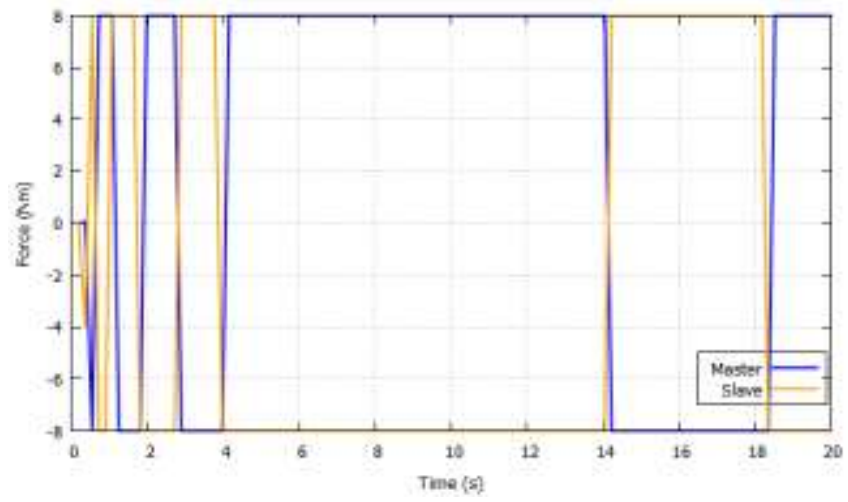

Fig. 18. Force of Master and Slave Arm vs. Time for $\omega_{n}=500$. 
All graphs from Fig. 10 to Fig. 18 illustrate the feedback in torque reading on the single joint of both master and slave system in various parameter values, ranging specify at value $\omega_{n}=1$ to $\omega_{n}=500$. The graph line formed in blue color represents the torque reading for the master robot, while the orange line shows the torque reading recorded on the slave robot. The preset maximum torque value for Joint0 in both articulated robots is $8 \mathrm{Nm}$. In Fig. 10 to Fig. 14, when force reading in master increased to $8 \mathrm{Nm}$ after being forced by the external manipulator, the reading value on the slave robot started to create series of progressive variations in the force reading. At this moment, the slave remains stationary from its initial position. This is because the value of gain $K_{p}$ and $K_{d}$ are relatively small and unfit to generate a working bilateral scheme. Although force exerted on master robot getting an increase for a brief period after the push, system on slave side considered the information feed from the other pair to be disregarded. The slave robot is unable to read the data that passes through, as reading is unstable and constantly changing. This situation also occurred after the external manipulator returned to its initial position and ceased pushing the master youBot arm ahead. Hence, torque readings for both master and slave are continually fluctuating and show no evidence of colliding or contacting with other external forces.

Whereas the force reading in the slave improved and matched the force reading recorded in the master's as shown in Fig. 15 to 18 . This is because, at $t=4 \mathrm{~s}$, the external arm began pushing the master youBot. After receiving information from its pair, the slave sensing the same applied force and move ahead as well for a certain period until obstructed by another block. Slave youBot instantly exerting a reaction force against the barrier and attempting to go forward. From the graph, the lines formed indicate that the master youBot arm applied its maximum torque of $+8 \mathrm{Nm}$ to go further, while the slave youBot arm attempted to withstand the greater force applied by an external obstacle, resulting in a value of $-8 \mathrm{Nm}$ in torque. The bigger gain values for the controllers demonstrate the connection between the magnitudes of the input and output signals at a steady state. For example, when the external manipulator returned to its position after $12 \mathrm{~s}$ and ceased its $8 \mathrm{Nm}$ push on the master robot, joint reading for both subsystems (master-slave robot) wavered and unstable. To improve the system stability, increasing the value of gain controller for both subsystems will cause the bilateral system to operate ideally and obey the bilateral law of the control system, as illustrated in Fig. 15 to Fig. 18 when the value of gain is respectively set at $K_{p}=2500$ and $K_{d}=100, K_{p}=10000$ and $K_{d}=200, K_{p}=40000$ and $K_{d}=400$ and $K_{p}=250000$ and $K_{d}=1000$. Thus, whenever the force reads on Joint0 of the master is $+8 \mathrm{Nm}$, Joint 0 on the slave will be giving a counter at $-8 \mathrm{Nm}$.

\section{B. DOB based for Position Control (Part B)}

For this experiment, position control is backed up by trials that demonstrate the DOB's capability to the bilateral system after being paired with the PD controller. Thus, graphs in Fig. 19 to Fig. 27 showed the position readings of both master-slave robots at specified Joint0.
All graphs from Fig. 19 to Fig. 27 illustrates the feedback in position reading on the single joint of both master and slave system in various parameter values, ranging specify at value $\omega_{n}=1$ to $\omega_{n}=500$. Graph line formed in blue color denotes the reference angle or step input, while the green line signifies the position reading logged on master robot and red line denotes the joint position recorded on slave robot. The preset maximum torque value for Joint 0 in both articulated robots is $8 \mathrm{Nm}$ and the position angle was initially set at $0^{\circ}$ for both pairs of manipulators. Reading for the position of the master reaches a maximum around $t>8.5 \mathrm{~s}$ and is maintained until 20s, looking from graphs in Fig. 18 to Fig. 23.

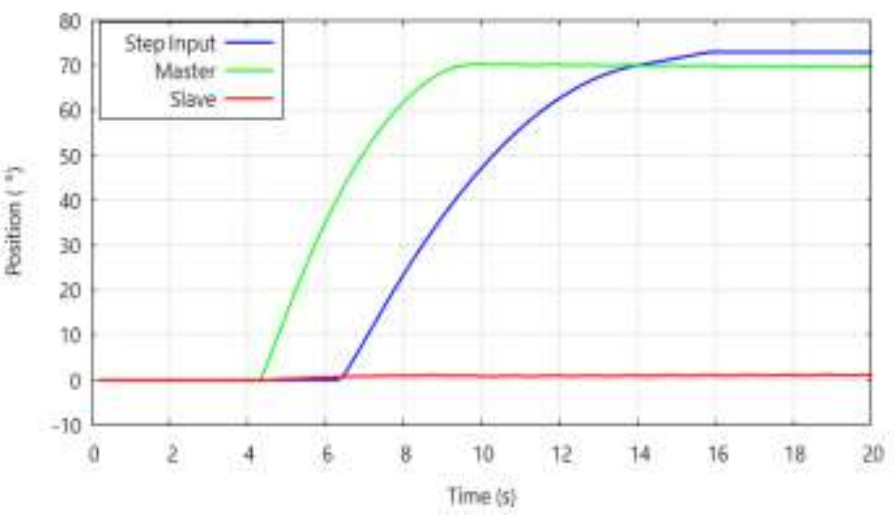

Fig. 19. Position of Master and Slave Arm vs. Time for $\omega n=1$.

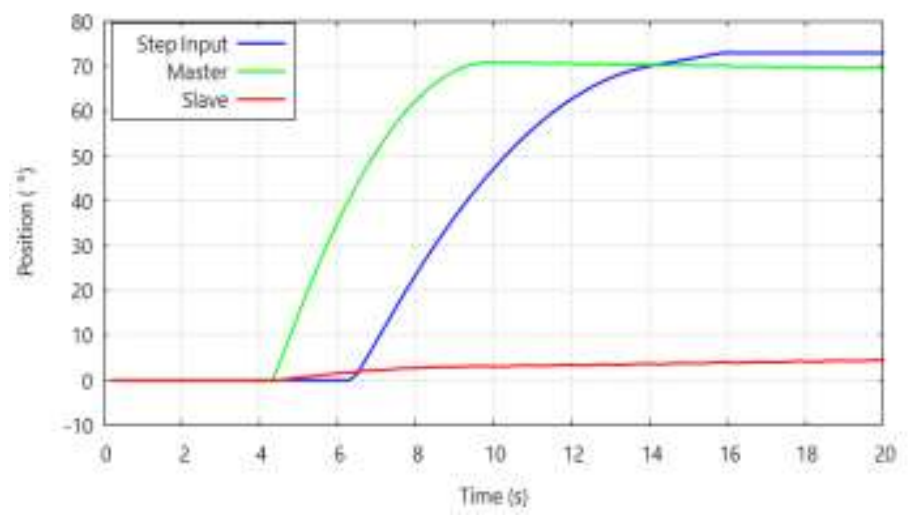

Fig. 20. Position of Master and Slave Arm vs. Time for $\omega n=2$.

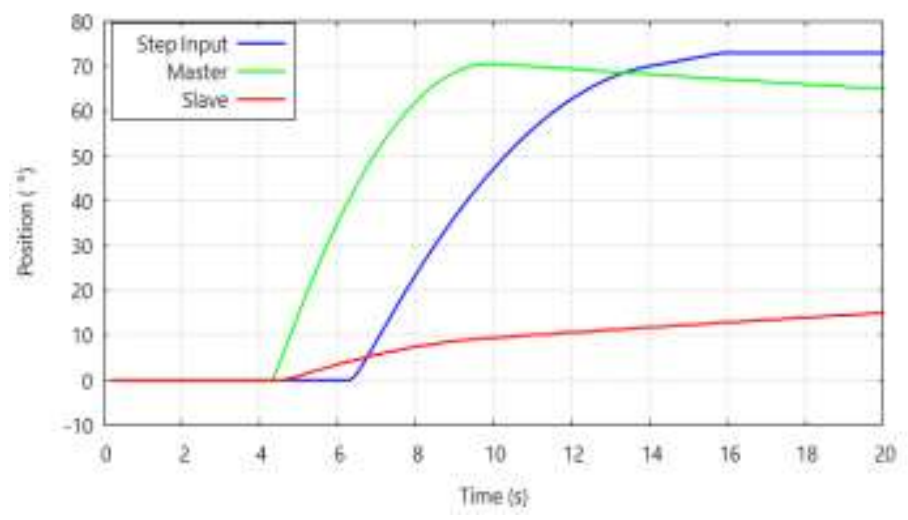

Fig. 21. Position of Master and Slave Arm vs. Time for $\omega n=5$. 


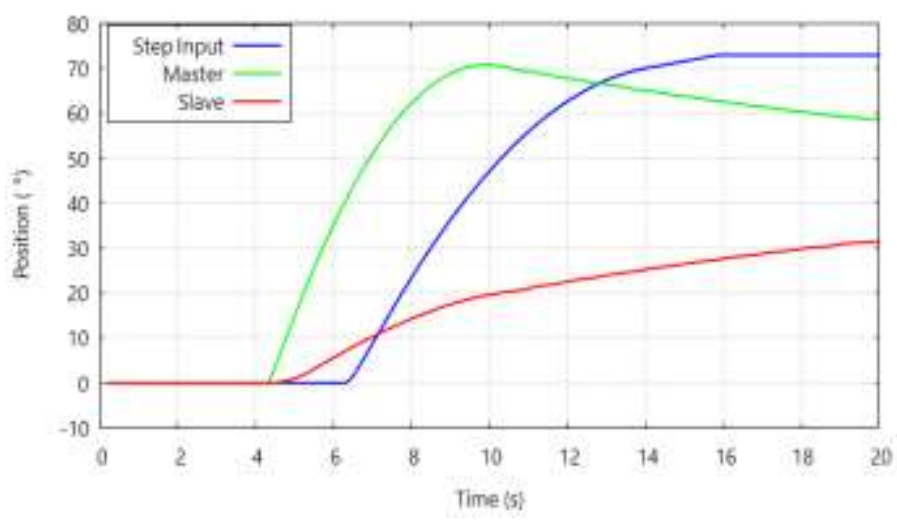

Fig. 22. Position of Master and Slave Arm vs. Time for $\omega n=10$.

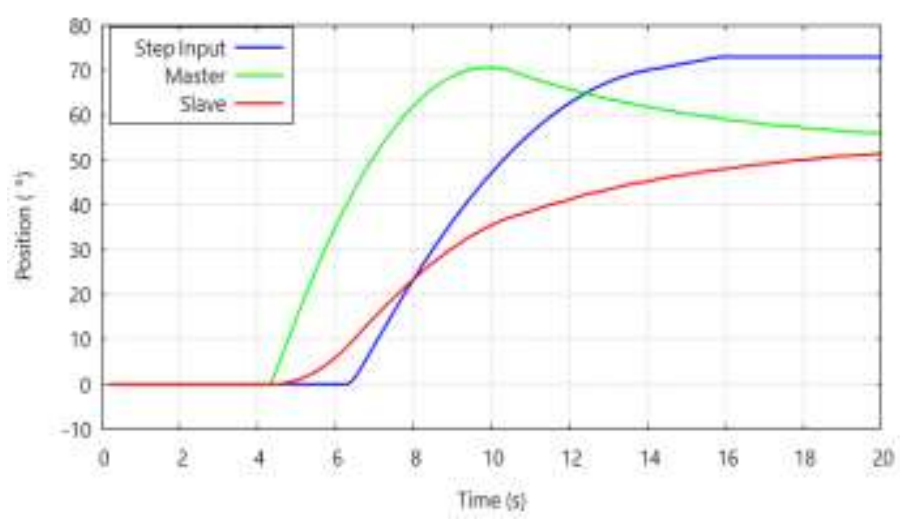

Fig. 23. Position of Master and Slave Arm vs. Time for $\omega n=20$.

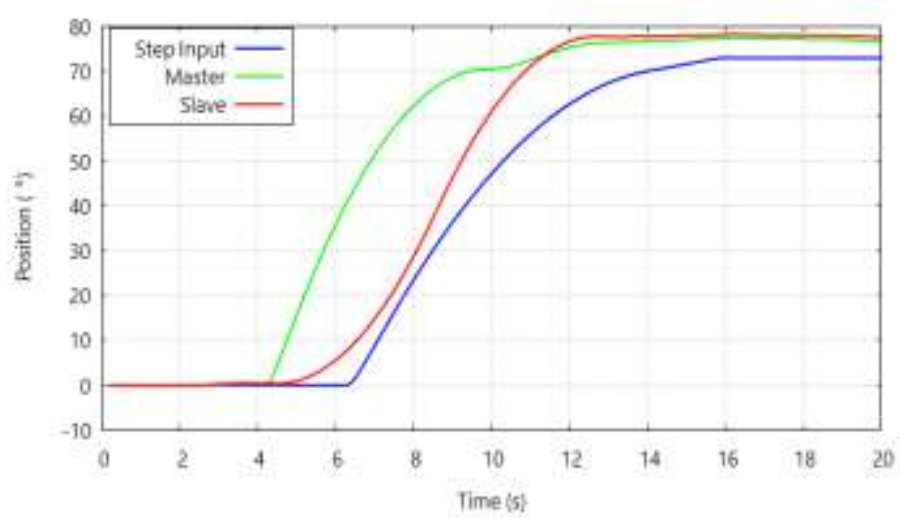

Fig. 24. Position of Master and Slave Arm vs. Time for $\omega n=50$.

Individually, the position of the slave achieved maximum position angle at $51^{\circ}$ in Fig. $23,20^{\circ}$ in Fig. $22,15^{\circ}$ in Fig. 21, and $5^{\circ}$ in Fig. 20. After $t>5 \mathrm{~s}$, the slave's position grew slightly but no distinct disparity with the first three graphs. On the other hand, at $t=9 \mathrm{~s}$, the graph in Fig. 19 to Fig. 23 showed that the position of the master topped across $70^{\circ}$ and roughly reached $80^{\circ}$ for Fig. 24. Different situations were observed from graphs 25, 26, and 27, respectively. Both robots swinging forth and back while steadily increase in their positions. It appears that the position readings at the master side continue to rise after an external force is exerted on the master robot.

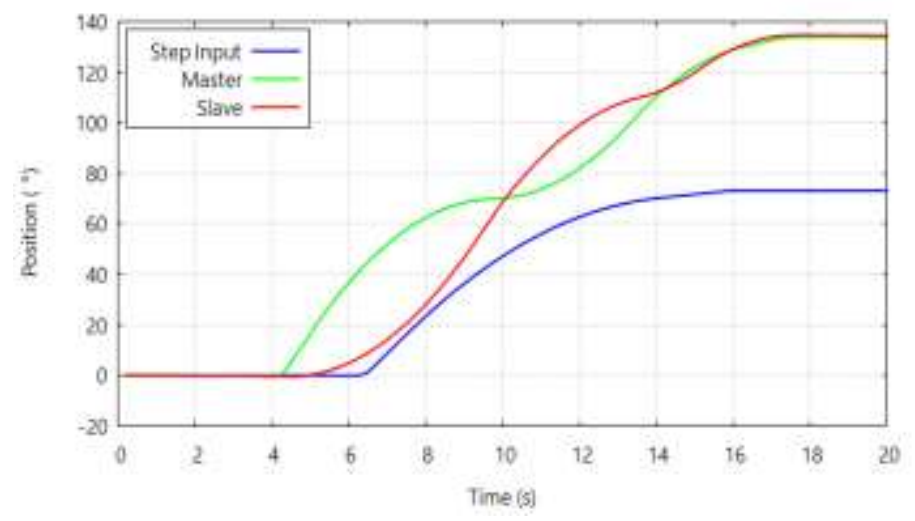

Fig. 25. Position of Master and Slave Arm vs. Time for $\omega n=100$.

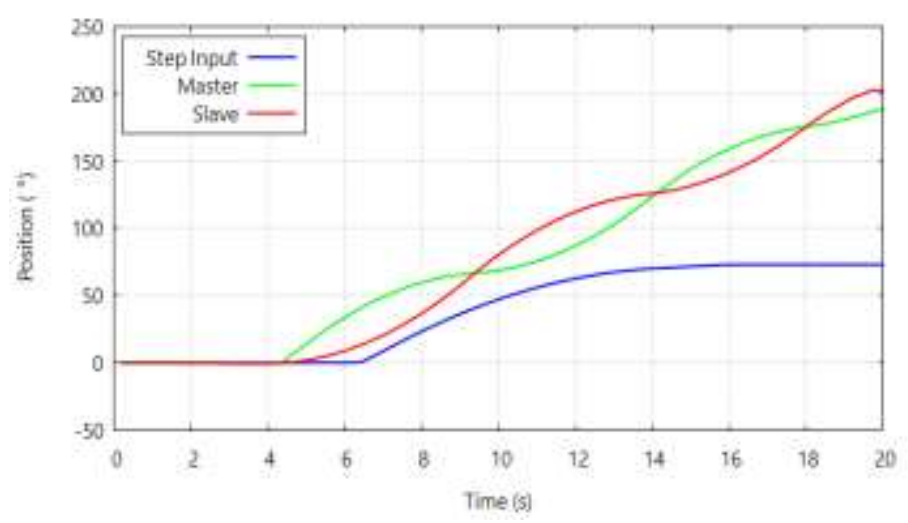

Fig. 26. Position of Master and Slave Arm vs. Time for $\omega n=200$.

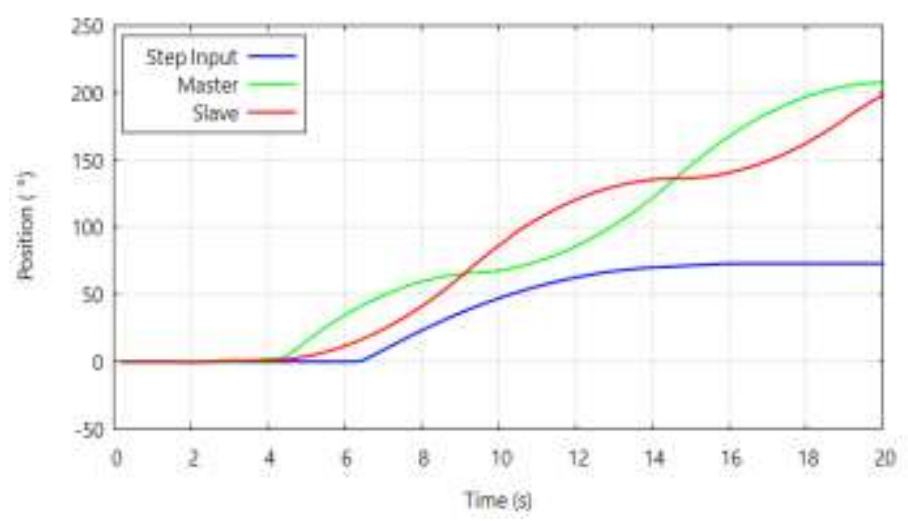

Fig. 27. Position of Master and Slave Arm vs. Time for $\omega_{n}=500$.

Meanwhile, the pattern of the graph in Fig. 19 to Fig. 21 shows that the angle position expands slightly as being compared to the master because the value of the PD controller is insufficient to reach an overshoot or settling time. Following the increased value of gain, the position angle of the slave robot extended and advanced, peaked at $78^{\circ}$ in maximum position with a stable condition as portrayed in Fig. 24 and $200^{\circ}$ in unstable condition, formed in each graph in Fig. 26 and 27. Simultaneously, the position angle for the master robot began to converge with the slave's position, as seen in Fig. 24 and Fig. 25. 
Reading of angle at Joint0 on slave robot according to Fig. 25 to Fig. 27 showed that it went further to catch up with the master's position, while the master robot is attempting to catch up with the slave's position as well. Delays between the two subsystems occurred during exchanging the data, which caused the angle to vary continuously. When $\omega_{n}=100$, both robots were able to achieve the same position at $137^{\circ}$ after fluctuating for about $13 \mathrm{~s}$. The position values of the master and slave exceeded the reference angle after the value of $\omega_{n}$ is set at 50 to 500 . Error value in position angle between master and slave and the reference angle is smallest, approximately $5^{\circ}$ as shown in Fig. 24 and largest $\left(-125^{\circ}\right)$ in Fig. 27. Table II summarizes the accuracy for values of $\omega_{n}$ in the stated controller.

TABLE II. ACCURACY FOR CONTROL SYSTEM WITH DOB

\begin{tabular}{|l|l|l|l|}
\hline$\omega_{n}$ & $K_{p}\left(\omega_{n}\right)^{2}$ & $K_{d}\left(2 \omega_{n}\right)$ & Accuracy $(\%)$ \\
\hline 1 & 1 & 2 & 1.78 \\
\hline 2 & 4 & 4 & 6.49 \\
\hline 5 & 25 & 10 & 23.09 \\
\hline 10 & 100 & 20 & 53.79 \\
\hline 20 & 400 & 40 & 91.82 \\
\hline 50 & 2500 & 100 & 98.94 \\
\hline 100 & 10000 & 200 & 99.54 \\
\hline 200 & 40000 & 400 & 92.66 \\
\hline 500 & 250000 & 1000 & 95.31 \\
\hline
\end{tabular}

Three systems have an accuracy of greater than $95 \%$. The system with $\omega_{n}=100$ has the best accuracy at $99.54 \%$, followed by the system with $\omega_{n}=50$ at $98.94 \%$ and the system with $\omega_{n}=500$ at $95.31 \%$. Although the last system acquired the highest accuracy, both robots swayed further before settling into the same position relative to one another. Meanwhile, there are two systems with an accuracy rate of less than $10 \%$. The system has an accuracy of $1.78 \%$ when $\omega_{n}=1$ while another system with precision of $6.49 \%$ when $\omega_{n}=2$. Conferring to graph shape in Fig. 24, the optimal design for a control system with DOB based for position control is $K_{p}=2500$ and $K_{d}=100$ since both bilateral master and slave robot reached the same position fixedly after $11 \mathrm{~s}$, has a smaller error in steady-state, and attained a critical damped. For force control, the optimal design for a control system is the value of $K_{p}=250000$ and $\mathrm{K}_{d}=1000$. Due to force readings recorded at Joint0 inside master and slave systems is indicated at zero for summation of torque and has the least feedback noise, as seen in Fig. 18.

\section{DISCUSSION AND CONCLUSION}

Tables III and IV summarized the best values for controllers and observations made from the analysis in simulation and graph patterns from all experiments.

Overall, this study successfully met all the objectives and was supported by two different experiments with a variety of different parameter sets to validate the system response. All results have been recorded and transformed into graphs to show the feedback pattern. The control system response with the proposed adaptive technique is also evaluated and discussed in form of accuracy, time delay, and settling time. It is shown that the adopted control technique as discussed by Jing et al., in [8] improves the stability and performance of the bilateral control system and is practical to apply in various systems including industrial robot simulations. The application of the observers by replacing the old-style force sensors on the robot control system has improved the dynamics inside the whole system, estimates the disturbance inside to improve the errors, and adjusting the input signal to achieve robust control motion, like the outcome of control system observed in [6].

TABLE III. SUMMARY FOR DOB IN COMMON MODE LAW

\begin{tabular}{|l|l|}
\hline \multicolumn{2}{|l|}{ Common Mode Law } \\
\hline Best values & $K_{p}=250000$ and $K_{d}=1000$ \\
\hline Worst values & $K_{p}=1$ and $K_{d}=2$ \\
\hline Force reading & $\begin{array}{l}\text { Total in force between master and slave are equal to } \\
\text { zero }\end{array}$ \\
\hline Force pattern & More vibrations, not stable \\
\hline
\end{tabular}

TABLE IV. SUMMARY FOR DOB IN DIFFERENTIAL MODE LAW

\begin{tabular}{|l|l|}
\hline Differential Mode Law \\
\hline Best values & $K_{p}=2500$ and $K_{d}=100$ \\
\hline Worst values & $K_{p}=250000$ and $K_{d}=1000$ \\
\hline Time delay & $+0.5 s$ \\
\hline Overshoot & $9.0 s$ to $9.5 s$ \\
\hline Accuracy & $98.94 \%$ \\
\hline Position reading & $\begin{array}{l}\text { Difference in total position between master and slave } \\
\text { are almost zero }\end{array}$ \\
\hline
\end{tabular}

For future work, another control tool called Reaction Force Observer (RFOB) could be introduced to the framework design of the bilateral control system to improve enhances the system's feedback and resilience. RFOB is known to have the ability to subtract the system uncertainties from the DOB input. It cancels out disturbance signals such as gravity forces, viscous damping friction, or Coriolis forces within both the internal system and internal motor located at the robot joint.

\section{ACKNOWLEDGMENT}

The authors would like to express their gratitude for the funds and support provided by Universiti Teknikal Malaysia Melaka (UTeM) under the Zamalah Scheme to conduct this study. Additionally, the authors would like to express appreciation to the Faculty of Electrical Engineering's Robotics and Industrial Automation Research Group.

\section{REFERENCES}

[1] B., Abeer, A. K., Hend, and A. S., Abdulmalik, "Haptics-Based Systems Characteristics, Classification, and Applications", USA: Advanced Methodologies and Technologies in Artificial Intelligence, Computer Simulation, and Human-Computer Interaction, 2019, pp.778-794.

[2] G., G. Shankar, M., Yaser, and Z., Kourosh, “An Application-Based Review of Haptics Technology", Robotics 2021, vol. 10, no. 29, 2021, pp. 1-18.

[3] N., Hiroshi, O., Kiyoshi, Y., Yuki, K. Naoki, M., Toshimasa, T., Akifumi, "Force Sensorless Fine Force Control Based on Notch-Type 
Friction-Free Disturbance Observers", IEEJ J. Ind. Appl., vol. 7, 2018, pp. 117-126.

[4] L., Xiangxing, Z., Guokun, Z., Jiaji, and W., Jiajin, "Sensorless Force Estimation of End-Effect Upper Limb Rehabilitation Robot System with Friction Compensation”, Int. J. Adv. Robot. Syst., vol., 16, 2019, pp. 111.

[5] A. R. Maouche and H. Meddahi, "A Fast Adaptive Artificial Neural Network Controller for Flexible Link Manipulators", Int. J. Adv. Comp. Sci. and App. (IJACSA), vol. 7, no. 1, 2016, pp. 298-308.
[6] E. Sariyildiz, and K. Ohnishi, "A Comparison Study for Force Sensor and Reaction Force Observer-based Robust Force Control Systems", Proc. IEEE 23rd Int. Con. Ind. Electron, 2014, pp. 1156-1161.

[7] L., Sichao, W., Lihui, and W., Vincent, "Sensorless Force Estimation for Industrial Robots Using Disturbance Observer and Neural Learning of Friction Approximation", Robot Comput. Integr. Manuf., vol. 71, 2021, pp. 1-11.

[8] B. Jing, H. Duan, J. Na, and S. Wang, "Unknown System Dynamics Estimator based Control for Bilateral Teleoperation Systems", 2021 IEEE 10th Data-Driven Control and Learning Systems Conf. (DDCLS), 2021, pp. 1552-1557. 